\title{
Calculation of the Critical Energy of the Power System with Protection Operation
}

\author{
Lin Jikeng Wang XuDong Z Zheng Weihong
}

\begin{abstract}
Whether a disturbance will trigger unexpected protection operation to exacerbate the normal operation state or even lead to the unstable state depends on the transient energy that the system gains during the fault period. The paper presents a new gradient projection approach to calculate the critical energy for transient stability assessment of the power system with the protection operation. The method takes the projection of the potential energy gradient on the key protection operation surface as search direction and iteratively searches for the critical energy at the surface; in order to let the optimal point be the feasible point, the generator angles are considered as control variables of power flow equation to solve network bus voltage and angle during the searching process. Examples show the convergence and precision of the new method proposed is much better than that of other methods, which demonstrates the validity and feasibility of the new method.
\end{abstract}

Keyword--power system, transient stability, protection, critical energy, gradient projection

\section{INTRODUCTION}

Voltages and currents often undergo significant excursions during the transient period following the clearing of a large disturbance. This can result in unexpected switching events, which often have a detrimental effect on system recovery, such as voltage collapse, large rotor swings between generators to result in distance protection tripping feeders and voltage dips. Therefore, it is very important to assess whether a disturbance will trigger unexpected protection operation to exacerbate the normal operation state or lead to the unstable state, and adjust protection and control schemes to avoid potential undesirable events.

${ }^{1}$ Ref. [1] proposes to use the relay margin as an index to assess the vulnerability of the line. Ref. [2] presents the sensitivity of line to be the index to identify the most vulnerable line that could be tripped out and therefore exacerbates the system stability. The method based on energy function is adopted in Ref [3] to assess out-of-step

\footnotetext{
1 This work was supported by National Science Foundation of P.R.China under the Grant: 50477036.

Lin Jikeng (Ph.D)works as associated professor at the School of Electrical Power and Automation, Tianjin University, Tianjin, P. R. China. E-mail:mejklin@126.com.

Wang Xudong is a master degree candidate of Tianjin University, Tianjin, P. R. China

Zheng Weihong (B. D) works as a senior engineering at the Dispatch Center of Tianjin Electric Power Corporation, Tianjin, P.R.China
}

protection operation. The method is later extended to assess the voltage dip and the operation of voltage protection [4]. A general framework to assess the transient stability with protection operation is presented in [5, 6]. Its main idea is to add the operation characteristic of the distance protection as the constraint condition of the dynamical system and educe the minimum potential energy of the protection surface of the key lines to be the system critical energy. Simulated annealing algorithm is proposed in Ref. [7] to calculate the minimum potential energy. In order to speedup the calculation, one-step approach and two-step approach based on Newton-Raphson algorithm are suggested in [8, 9]. The method adopted in Ref. [7] is stochastic, which has the good convergence; however, its calculation time is usually too long and uncertain. Although the calculation speed is fast for the method in the Ref. [8, 9], its convergence is not satisfactory, due to the fact that the system is non-convex .

This paper presents a method based on gradient-projection to calculate the minimum potential energy of the key protection operation surface of the key line. The test results of the samples demonstrate that the method has advantages in both convergence and accuracy over the methods proposed in the paper $[7,8]$.

\section{POWER SYSTEM MODEL AND ENERGY FUNCTION}

\section{A. Power System Model}

Assume that there are $n_{g}$ synchronous generators and $n_{l}$ load nodes in the system. Then the number of the extended network nodes including generator inner nodes is $n=n_{g}+n_{l}$, where $1,2, \ldots, n_{g}$ stand for the generator inner nodes and $n_{g}+1, \ldots, n$ stand for the load nodes. The load model is the static state model. The branch resistance is ignored. The system equations in COI reference are as follows:

$$
\begin{aligned}
& \dot{\theta}_{i}=\tilde{\omega}_{i} \\
& M_{i} \dot{\tilde{\omega}}_{i}=P_{M_{i}}-\sum_{j=1, j \neq i}^{n} B_{i j, j} V_{j} V_{i} \sin \theta_{i j}-\frac{M_{i}}{M_{T}} P_{C O I} \\
& P_{i}\left(V_{i}\right)+\sum_{\substack{j=1 \\
j \neq i}}^{n} B_{i j} V_{i} V_{j} \sin \theta_{i j}=0 \\
& Q_{i}\left(V_{i}\right)-\sum_{\substack{j=1 \\
j \neq i}}^{n} B_{i j} V_{i} V_{j} \cos \theta_{i j}-B_{i i} V_{i}^{2}=0
\end{aligned}
$$

Where 
Proceedings of the International MultiConference of Engineers and Computer Scientists 2008 Vol II IMECS 2008, 19-21 March, 2008, Hong Kong

$M_{T}=\sum_{i=1}^{n_{g}} M_{i} \quad P_{C O I}=\sum_{i=1}^{n_{g}}\left(P_{M_{i}}-\sum_{j=1}^{n} B_{i j} V_{i} V_{j} \sin \theta_{i j}\right)$

$V_{i}$ is the bus voltage magnitude, $\theta_{i}$ is the bus angle, and $M_{i}$ is the moment of inertia of the ith generator.

\section{B. Energy Function}

For the system (1)-(3), the energy function is:

$$
V=V_{K E}+V_{P E}
$$

Where

$$
\begin{gathered}
V_{K E}=\frac{1}{2} \sum_{i=1}^{n_{g}} M_{i} \tilde{\omega}_{i} \\
V_{P E}=-\sum_{i=1}^{n_{g}} P_{m_{i}}\left(\theta_{i}-\theta_{i}^{s}\right)+\sum_{i=1+n_{g}}^{n} \int_{t_{0}}^{t} P_{i}\left(V_{i}\right) \dot{\theta} d t \\
+\sum_{i=1+n_{g}}^{n} \int_{V_{i}^{s}}^{V_{i}} \frac{Q_{i}\left(V_{i}\right)}{V_{i}} d V_{i}-\frac{1}{2} \sum_{i=1+n_{g}}^{n} B_{i i}\left(V_{i}^{2}-V_{i}^{S 2}\right) \\
+\sum_{i=1}^{n-1} \sum_{j=i+1}^{n} B_{i j}\left(V_{i} V_{j} \cos \theta_{i j}-V_{i}^{s} V_{j}^{s} \cos \theta_{i j}^{s}\right)
\end{gathered}
$$

$V_{K E}$ is the kinetic energy, $V_{P E}$ is the potential energy.

\section{THE CRITICAL ENERGY FOR THE ASSESSMENT OF TRANSIENT STABILITY OF POWER SYSTEM WITH THE PROTECTION OPERATION}

The energy function method is based on the comparison of the energy acquired by the system during the fault period and the critical energy to assess system stability. For the power system with the protection operation, the critical energy should be the energy that might not induce the key protection to trip the key line[6]. If the system energy acquired during the fault period is less than the critical energy, there is no enough energy to induce any protection operation. And the possibility for the system to be instable due to the unexpected protection operation is completely eliminated.

It is worth noting that only distance protection is considered in the paper.

A. The Protection Operation Characteristic

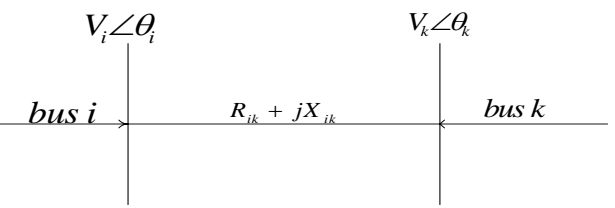

Fig. 1 the line between bus $\mathrm{i}$ and bus $\mathrm{k}$

The distance protection compares the impedance measured by the relay with the setting value and determines whether it should trip the line. For the line as Fig.1, the impedance measured at bus $i$ is:

$$
z_{i k}\left(\theta_{i}, \theta_{k}, V_{i}, V_{k}\right)=\frac{V_{i} \angle \theta_{i}}{\frac{V_{i} \angle \theta_{i}-V_{k} \angle \theta_{k}}{R_{i k}+j X_{i k}}}
$$

Where $V_{i}$ and $\theta_{i}, V_{k}$ and $\theta_{k}$ are the voltage magnitude and angle at bus $\mathrm{i}$ and at bus $\mathrm{k}$, respectively.
$R_{i k}$ and $X_{i k}$ are the feeder resistance and reactance, respectively. The operation characteristic of directional distance protection could be shown as Fig.2.

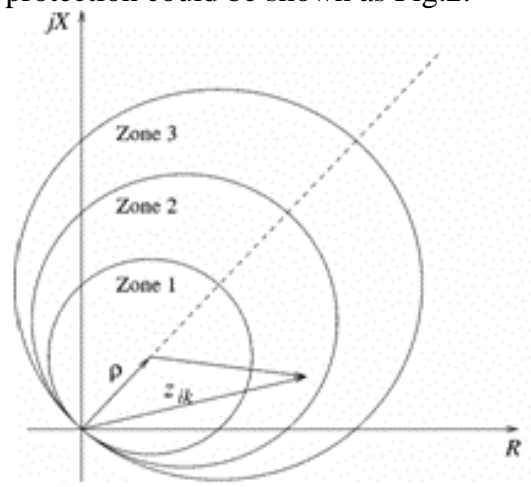

Fig. 2 the operation characteristic of directional distance protection The relay at bus i for the line ik will operate if

$$
\left|z_{i k}-\rho\right| \leq|\rho|
$$

Where

$$
\rho=\frac{\beta}{2}\left(R_{i k}+j X_{i k}\right)
$$

is the center of the mho circle in the complex plane. Each circle in Fig. 2 corresponds to a different value of $\beta$ : $\beta=0.8$ for zone $1, \beta=1.2$ for zone $2, \beta=1.6$ for zone 3. By substituting equations (7)、(9) into equation (8) , it can be obtained that:

$$
\left|\frac{v_{i} \angle \delta_{i}}{v_{i} \angle \delta_{i}-v_{k} \angle \delta_{k}}-\frac{\beta}{2}\right| \leq \frac{\beta}{2}
$$

Let:

$$
\left.w\left(\theta_{i}, \theta_{k}, V_{i}, V_{k} ; \beta\right)=\mid \frac{2 V_{i} \angle \theta_{i}}{V_{i} \angle \theta_{i}-V_{k} \angle \theta_{k}}-\beta\right) \mid
$$

Then the operation equation of the distance protection is:

$$
w\left(\theta_{i}, \theta_{k}, V_{i}, V_{k}, \beta\right) \leq \beta
$$

And the relevant operation region is:

$$
S_{i}=\left\{(x, y)\left|H_{i k}(y)=\right| \frac{2 V_{i} \angle \theta_{i}}{V_{i} \angle \theta_{i}-V_{k} \angle \theta_{k}}-\beta \mid-\beta \leq 0\right\}
$$

Where, $H_{i k}(x, y)=0$ is the boundary of the operation region, which could also be expressed as : $\bar{S}_{i}=\left\{(x, y) \mid H_{i k}(x, y)=0\right\}$. $\bar{S}_{i}$ denotes protection operation surface or the protection surface.

\section{B. The Critical Energy With The Protection}

For the system with protection operation, the critical energy is the minimum energy acquired by the system to lead the post-fault trajectory to be tangential to the key protection surface [6-9]. So the estimation of this critical energy can be obtained by solving the minimization problem:

$$
\begin{aligned}
& V_{c r}=\min _{(x, y) \in \bar{S}_{i}} V_{P E}(x, y) \\
& \text { s.t. } \quad g(x, y)=0
\end{aligned}
$$

The equation (11) can be rewritten as: 
Proceedings of the International MultiConference of Engineers and Computer Scientists 2008 Vol II

IMECS 2008, 19-21 March, 2008, Hong Kong

$$
\begin{array}{ll}
V_{c r}=\min _{x} V_{P E}(x, y) \\
\text { s.t. } & g(x, y)=0 \\
& H^{*}(x, y)=0
\end{array}
$$

Where, $H^{*}(x, y)=0$ is the key protection operation surface corresponding to the key line, superscript $*$ denotes the key protection; $g(x, y)=0$ is the power flow constraint equation.

\section{GRADIENT PROJECTION ALGORITHM}

To solve equation (12), one-step approach and the two-step approach based on Newton-Raphson algorithm are proposed in $[8,9]$. Because of the non-convex characteristic, their convergences are not satisfactory. Therefore, this paper proposes a new gradient projection algorithm to calculate the critical energy, which has good convergence. To solve equation (12) by gradient method, the key issue is to how to process the constraint conditions. The strategy adopted in the paper is to let all the feasible solutions be at the surface of the key protection operation equation all the time, and the projection of the potential energy at the protection operation surface to be the search direction to iteratively search for the critical energy. The power flow constraint equation is satisfied by using the new generators' $\theta_{i}\left(i=1,2, \ldots, n_{g}\right)$ to solve the power flow equations to obtain the corresponding $V_{i}, \theta_{i}\left(i=n_{g}+1, \ldots, n\right)$.

The gradient projection on the tangent plane of the protection operation surface is shown in Fig.3, where $\vec{S}=(l, m, n)$ is the negative gradient direction of the object function $V_{P E}(x, y) ; \pi$ is the tangent plane of the protection operation surface; $P$ is the intersection point of $\vec{S}$ with the protection operation surface; $\vec{n}=(A, B, C)$ is the vertical vector of the tangent plane $\pi ; \varphi$ is the angle between $\vec{S}$ and the tangent plane $\pi ; \quad \overrightarrow{P Q}$ is the projection of $\vec{S}$ on the tangent plane $\pi$.

$\sin \varphi=\cos \left(\frac{\pi}{2}-\varphi\right)=\frac{\vec{S} \cdot \vec{n}}{|\vec{S}||\vec{n}|}=\frac{A l+B m+C n}{\sqrt{A^{2}+B^{2}+C^{2}} \sqrt{l^{2}+m^{2}+n^{2}}}$

The projection direction is:

$$
d \equiv \overrightarrow{P Q}=\overrightarrow{S^{0}}-\overrightarrow{n^{0}} \sin \varphi
$$

Where $\vec{S}^{0}$ and $\vec{n}^{0}$ are the unit vector of $\vec{S}$ and $\vec{n}$ respectively.

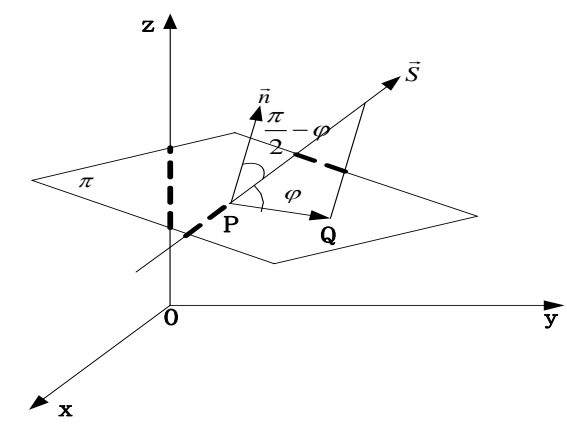

Fig. 3 the projection of the negative gradient of $V_{P E}(x, y)$ at the tangent plane of the protection operation surface

The negative gradient direction of the object function $V_{P E}(x, y)$ is orthogonal to the tangent plane of the protection operation surface when the length of $\overrightarrow{P Q}$ is zero. Then the minimum potential energy is found.

The optimization process of the gradient approach is is as follows:

(1) $\mathrm{k}=0$;

(2) The negative gradient of the potential energy $V_{P E}$ is computed. Since $\nabla_{y} V_{P E}=g(x, y)=0$, the negative gradient of the potential energy at $x^{k}$ is:

$$
\vec{S}=-\left.\nabla_{\chi} V_{P E}\right|_{x=x^{k}}=\left.\left[-\frac{\partial V_{P E}}{\partial x_{1}},-\frac{\partial V_{P E}}{\partial x_{2}}, \cdots,-\frac{\partial V_{P E}}{\partial x_{n_{g}}}\right]^{T}\right|_{x=\chi^{k}}
$$

Where $\left.\frac{\partial V_{P E}}{\partial x_{i}}\right|_{x_{i}=x_{i}^{k}}=\left.\frac{\partial V_{P E}}{\partial \theta_{i}}\right|_{\substack{\theta_{i}=\theta_{i}^{k} \\ V_{i}=V_{i}^{k}}}=-P_{m_{i}}+\sum_{j=1}^{n_{l}} B_{i j} V_{i}^{k} V_{j}^{k} \sin \theta_{i j}^{k}$

(3) The vertical vector of the tangent plane of the protection surface is computed. since

$$
\left\{\begin{array}{l}
g(x, y)=0 \\
\frac{\partial H^{*}(y)}{\partial x}=\frac{\partial H^{*}(y)}{\partial y} \square \frac{\partial y}{\partial x}
\end{array}\right.
$$

It could be obtained that:

$$
\frac{\partial H^{*}(y)}{\partial x}=-\frac{\partial H^{*}(y)}{\partial y} \frac{\partial g^{-1}}{\partial y} \frac{\partial g}{\partial x}
$$

Then

$$
\begin{gathered}
\vec{n}=\left.\nabla_{x} H^{*}(y)\right|_{x=x^{k}}=-\left.\nabla_{y} H^{*}(y) \sqcap g_{y}^{-1}(x, y) g_{x}(x, y)\right|_{\substack{x=x^{k} \\
y=y^{k}}} \text { Where }\left.\nabla_{y} H^{*}(y)\right|_{y=y^{k}}=\left[\frac{\partial H^{*}(y)}{\partial \theta_{1+n_{g}}}, \cdots, \frac{\partial H^{*}(y)}{\partial \theta_{n}}, \frac{\partial H^{*}(y)}{\partial V_{1+n_{g}}}, \cdots, \frac{\partial H^{*}(y)}{\partial V_{n}}\right]_{\substack{\theta-y^{*+1} \\
v=v^{k+1}}}^{T}
\end{gathered}
$$

$g_{y}\left(x^{k}, y^{k}\right)$, is the partial derivative matrix of the power flow relative to the bus voltage and angle of the network; $g_{x}(x, y)$ is the partial derivative matrix of the power flow relative to the generator angles.

(4) The projection of the negative gradient of the potential energy $V_{P E}$ at the tangent plane of the protection operation surface $-d$ could be obtained by substituting (15)、(16) into (13) and (14).

(5) If $d=0$, the minimum potential energy is obtained, and the potential energy at the state is the 
Proceedings of the International MultiConference of Engineers and Computer Scientists 2008 Vol II IMECS 2008, 19-21 March, 2008, Hong Kong

critical energy; Otherwise, $x^{\prime k+1}$ is obtained by one dimension optimization search on the direction $d$.

(6) The potential energy at $x^{\prime k+1}$ must be less than that at $x^{k}$; however, $x^{\prime k+1}$ is not sure to lie on the protection operation surface. Connecting $x^{\prime k+1}$ to the post-fault SEP with a line, the intersection point of the line with the protection surface is $x^{k+1}$. And the corresponding $y^{k+1}$ is achieved by solve the power flow equation: $g\left(x^{k+1}, y^{k+1}\right)=0$.

(7) Go to step (2).

\section{EXAMPLE}

The above method is tested with WSCC system and New England system, and the results are compared with those obtained by the two-step approach, which is much better than the one -step approach[8-9]..

A. WSCC System

The diagram of WSCC system is shown in Fig.4. The three cases of the three phases short-circuit fault considered occur at bus 4 of the line $4-3$, at bus 5 of the line $5-1$ and at bus 5 of the line $5-2$, respectively.

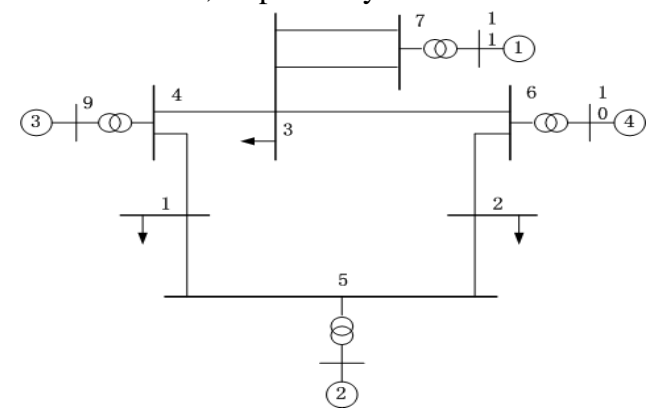

Fig.4 diagram of the WSCC system

\section{B. New England System}

The diagram of WSCC system is shown in Fig.4. The three cases of the three phases short-circuit fault considered occur at bus 16 of the line $16-21$, at bus 26 of the line 26-28, at bus 17 of the line 16-17,respectively.

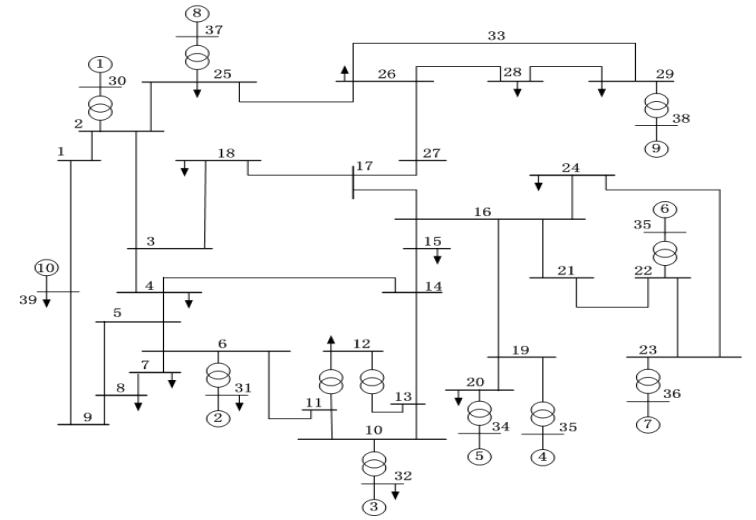

Fig. 5 the diagram of the New England system

All the faulted lines are cleared at 0.1 s and there are no re-closing following the faults. The critical energy for the three faults using two-step approach and the gradient projection method for WSCC system and New England system are shown in Tab.1 and Tab.2, with $\beta=0.85$. In the two tables, the protections of the key lines are the key protections. It could be seen from Tab.1 Tab. 2 that, most of the cases by the two-step approach could not be convergent except for two cases; On the contrary, all the cases could be convergent by the gradient projection method. The critical energy for most cases obtained by the gradient projection method is very close to the actual critical energy. It can also be seen that the critical energy with the protection operation is smaller than that without protection operation, however, the critical energy with the protection operation completely gets rid of the possibility to lead the system from stable state to the unstable state induced by the protection malfunction due to the system swing.

\section{CONCLUSION}

A new method based on the gradient projection to calculate the critical energy for the assessment of transient stability of power system with protection operation is proposed. The test results based on two example systems demonstrate that the new method has significant advantages over the two-step approach in term of iteration convergence and computation accuracy. The critical energy with the protection is less than that without the protection, and makes system operate in a more conservative state. However, it completely avoids the unexpected switching event due to system swing, which might lead the system from stable state to unstable state.

Table 1:the critical energy with the protection operation at $\beta=0.85$ for WSCC

\begin{tabular}{|c|c|c|c|c|c|}
\hline $\begin{array}{c}\text { Fault } \\
\text { line }\end{array}$ & Key line & $\begin{array}{c}\text { Two step } \\
\text { approach }\end{array}$ & $\begin{array}{c}\text { Gradient } \\
\text { projection }\end{array}$ & $\begin{array}{c}\text { Actual critical energy with } \\
\text { protection }\end{array}$ & $\begin{array}{c}\text { Critical energy } \\
\text { without protection }\end{array}$ \\
\hline $4-3$ & $1-4$ & & 2.583 & $(2.57 \sim 2.61)$ & 3.79 \\
\hline $5-1$ & $2-6$ & & 3.992 & $(4.02 \sim 4.06)$ & 4.34 \\
\hline $5-2$ & $1-4$ & & 4.378 & $(4.32 \sim 4.37)$ & 4.70 \\
\hline
\end{tabular}


Proceedings of the International MultiConference of Engineers and Computer Scientists 2008 Vol II IMECS 2008, 19-21 March, 2008, Hong Kong

Table 2: the critical energy with the protection operation at $\beta=0.85$ for New England system

\begin{tabular}{|c|c|c|c|c|c|}
\hline Fault line & Key line & $\begin{array}{c}\text { Two step } \\
\text { approach }\end{array}$ & $\begin{array}{c}\text { Gradient } \\
\text { projection }\end{array}$ & $\begin{array}{c}\text { Actual critical energy } \\
\text { with protection }\end{array}$ & $\begin{array}{c}\text { Critical energy } \\
\text { without protection }\end{array}$ \\
\hline $16-21$ & $23-24$ & & 15.094 & $(19.85 \sim 20.13)$ & 32.07 \\
\hline $26-28$ & $26-29$ & 8.535 & 8.534 & $(7.90 \sim 8.01)$ & 10.94 \\
\hline $16-17$ & $14-15$ & 19.782 & 19.781 & $(18.46 \sim 18.76)$ & 23.61 \\
\hline
\end{tabular}

\section{REFERENCE}

[1] F. Dobraca, M. A. Pai and P. W. Sauer, Relay margins as a tool for dynamical security analysis, International Journal of Electrical Power and Energy Systems, 1990, Vol.12, No. 4, PP.226-234

[2] S.A.Soman and Tony B.Nguyen, Analysis of angle stability problems: a transmission protection system perspective, IEEE Trans. on Power Delivery, 2004, vol.19, no.3:1024-1033

[3] V. Vttal, T. Oh and A. A. Fouad, Apparent impedance correlation of transient energy function margin and time simulation, IEEE Trans. On Power Systems, 1988, Vol.3, No.2, PP:455-462

[4] A. Debs, Voltage dip at maximum angular swing in the context of direct stability analysis, IEEE Trans. On Power Systems, 1990, Vol. 5, No.4, PP:1497-1502

[5] K. L. Praprost and K. A. Loparo, A stability theory for constrained dynamic systems with applications to electric power systems, IEEE Trans. On Automatic Control, 1996, Vol. 41, No. 11, PP:1605-1617

[6] Chaman Sigh and I. A. Hiskens, Direct Assessment of Protection Operation and Non-Viable Transients, IEEE Trans. On Power Systems, 2001, Vol. 16, No. 3, PP:427-434

[7] Chaman Singh, I. A. Hiskends and P. R. Kumar, Application of simulated annealing to power system protection assessment, Proceedings of the $37^{\text {th }}$ Conference on Decision and Control, Tampa, FL, 1998

[8] Chaman Singh, I. A. Hiskens, Energy Function Optimization for Power System Protection Assessment, in Proceedings Australasian Universities Power Engineering Conference, Darwin, Australia, 1999

[9] Chaman Singh and I. A. Hiskends, Energy function assessment of protection operation, in Proceedings Australasian Universities Power Engineering Conference, Hobart, Australia, 1998P.

[10] Kundur ; edited by Neal J. Balu, Mark G. Lauby, Power system stability and control, Publisher: McGraw-Hill Professional, ISBN: 007035958X,1994 